\title{
Impact of Exposure to Animals During Childhood on Clinical Features in Schizophrenia
}

\author{
Resident Hela Maktouf, Dr Amine Larnaout, Dr Amina Aissa, Dr Amira Khelifa, Dr \\ Hanene Ben Ammar, Pr Zouhair El Hechmy, Razi Hospital, Tunisia.
}

Introduction: In severe mental illnesses, animals are often used as a rehabilitative and therapeutic tool, especially in animalassisted therapies $(1,2)$. However, its impact on schizophrenia has not seen further investigation.

The aim of this study is to investigate the effect of an early exposure to animals on the symptoms of the disease.

Method: In a cross-sectional study that included 105 patients with schizophrenia, a clinical evaluation, using PANSS and Calgary scales, was conducted. Patients were divided into two groups, depending on whether or not they had a pet at home until the age of 12. A comparison study has been made between these two groups.

Results: The results showed that 73 patients had one or more animals at home until the age of 12 and 32 patients did not. Significant differences were observed between the two groups in two items: Lack of conversational spontaneity and fluidity $(p=0.02)$ and difficulties in abstraction $(p=0.029)$. No significant differences were found in PANSS and Calgary other items.

Results

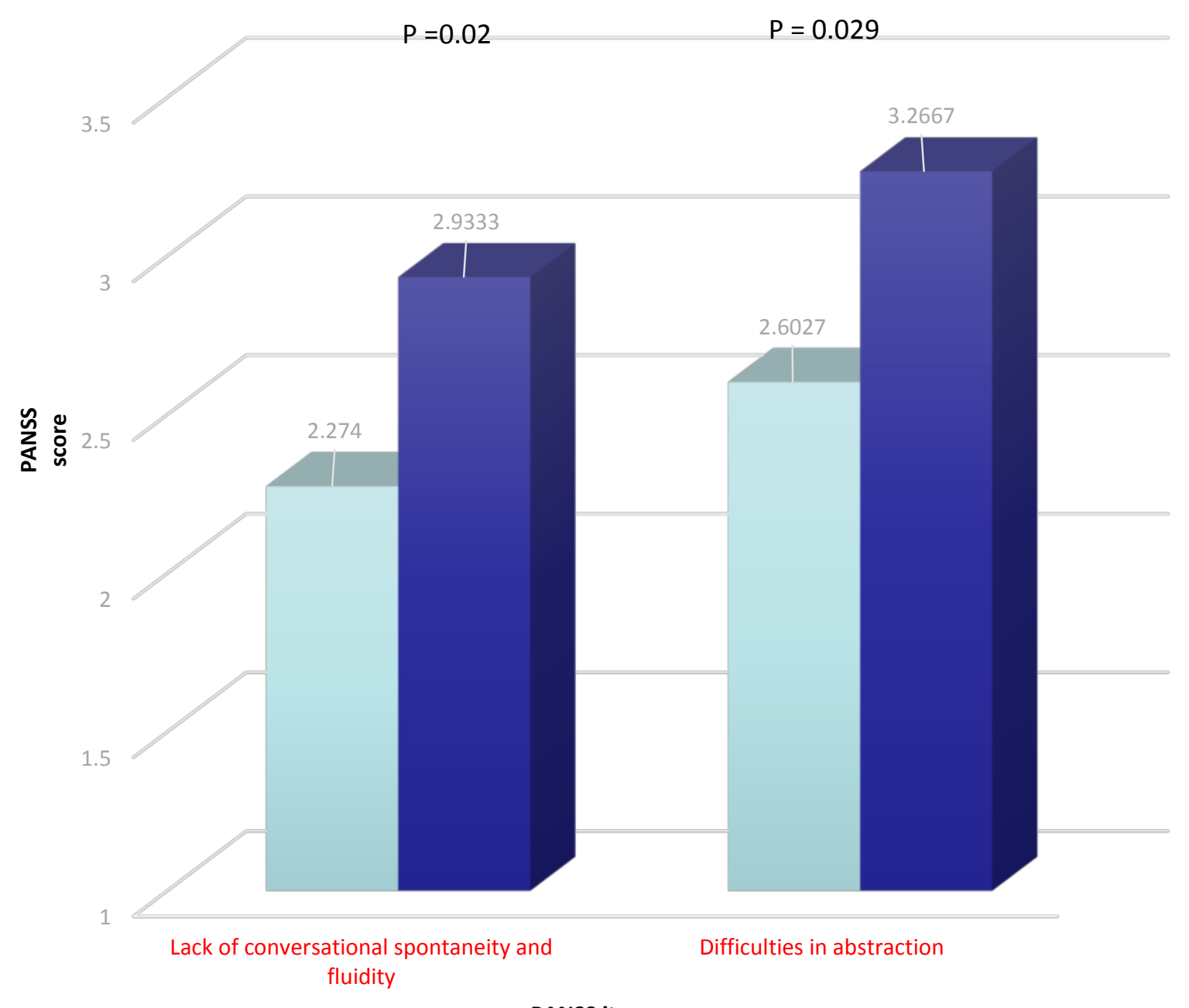

PANSS items
Discussion: As mentioned earlier, only etiological and therapeutic relationships were found between exposure to animals and schizophrenia disease $(1,2)$.

In our study, we found a strong correlation on the preventive effect of animals on patients with severe forms of schizophrenia . We hypothesize that having an animal before the age of 12 in patients with schizophrenia, may contribute to decrease anxiety in social relationships and lead to easier communication with others, through combating the low sensory and affective arousal levels (3). It may also alleviate social skills.

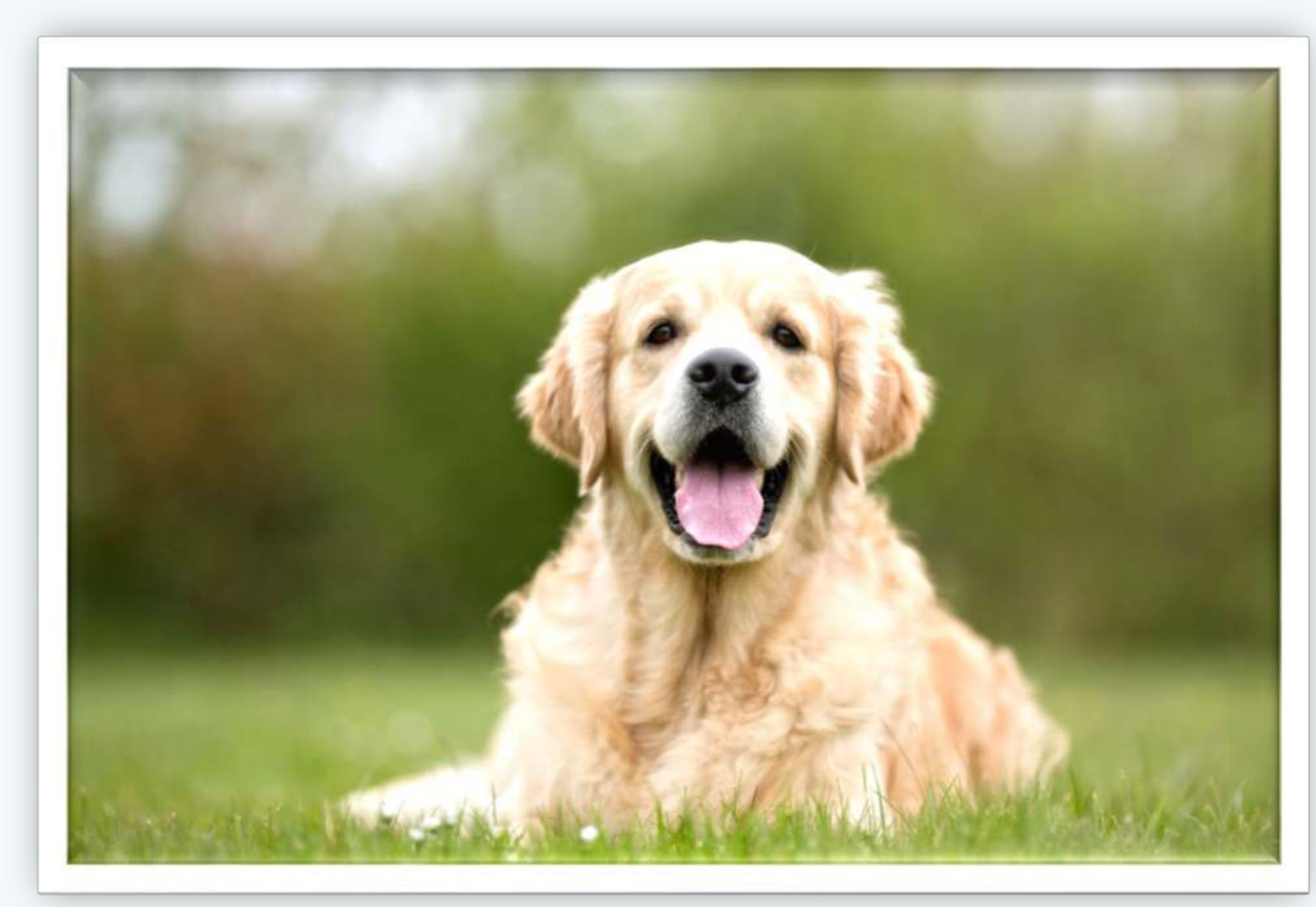

Conclusion: for patients with schizophrenia, having an animal from an early age leads to less severe symptoms of the disease. Further controlled studies are needed to confirm this and for more accurate definition of the complex link between schizophrenia and exposure to animals at an early age. This could benefit research on Ultra High Risk patients.

\section{References:}

1) Animal-assisted therapy with farm animals for persons with psychiatric disorders: effects on self-efficacy, coping ability and quality of life, a randomized controlled trial Bente Berget, Øivind Ekeberg and Bjarne O Braastad.

2) Animal-assisted therapy for schizophrenia and related disorders: A systematic review Emma L. Hawkins, Roxanne D. Hawkins, Martin Dennis, Joanne M. Williams, Stephen M. Lawrie.

3) Brief Report: Pet.Facilitated Therapy With Autistic Children Laurel A. Redefer and Joan F. Goodman Graduate School of Education, University of Pennsylvania. 